\title{
Solubilidade do metanol, etanol e isopropanol em óleos vegetais a diferentes temperaturas e pressão atmosférica
}

\author{
Methanol, ethanol and isopropanol solubility in vegetable oils at different temperatures \\ and atmospheric pressure
}

\author{
Alessandro Cazonatto Galvão, Regina Boschi, Karollina Aparecida Coelho, Diego Cadaval Machado, \\ Vanessa Zuqui, Weber da Silva Robazza \\ Departamento de Engenharia de Alimentos, Universidade do Estado de Santa Catarina - UDESC, \\ Pinhalzinho - Santa Catarina - Brasil \\ alessandro.galvao@udesc.br
}

\begin{abstract}
Resumo
O estudo da solubilidade de alcoóis em diferentes óleos vegetais é importante para processos industriais como, por exemplo, refino do óleo bruto, extração de óleos vegetais e processos de produção de biodiesel. Os resultados foram obtidos a partir de estudo do equilíbrio líquido-líquido utilizando célula de equilíbrio com agitação e termostatização. Os óleos vegetais escolhidos para o estudo foram óleo de canola, óleo de girassol, óleo de milho e óleo de soja. Os estudos foram conduzidos na faixa de 293,15 K a 333,15 K para os solventes metanol e etanol e na faixa de 290,15 Ka 296,15 K para o isopropanol. Observou-se um aumento da solubilidade dos alcoóis nos óleos vegetais com o aumento da temperatura. Observou-se também um aumento da solubilidade dos alcoóis nos óleos vegetais com o aumento da cadeia carbônica do álcool.
\end{abstract}

Palavras-chave: solubilidade, óleos vegetais, solventes

\begin{abstract}
The study of alcohols solubility in different vegetable oils is important for industrial processes, such as, refining of oil, extraction of oil and biodiesel production. The experimental measurements were performed in a liquid-liquid equilibrium cell under constant temperature and stirring. The solubility of methanol, ethanol and isopropanol was investigated in refined oils of soybean, corn, sunflower and rapeseed. Methanol and ethanol solubility were analysed in the temperature range between $293.15 \mathrm{~K}$ and $333.15 \mathrm{~K}$. Isopropanol solubility was analysed in the temperature range between $290.15 \mathrm{~K}$ and $296.15 \mathrm{~K}$. It was observed an increase of solubility with the increase of temperature and with the increase of alcohol chain length.
\end{abstract}

Keywords: solubility, vegetable oils, solvents 


\section{Introdução}

Os óleos vegetais são um dos principais produtos extraídos de sementes oleaginosas (REDA e CARNEIRO, 2007). No Brasil, são consumidos anualmente cerca de 3,72 milhões de toneladas de óleos vegetais, com $84 \%$ utilizados na alimentação e aproximadamente $16 \%$ para fins industriais. Os óleos vegetais são constituídos principalmente de triglicerídeos (95-98\%) e uma mistura de componentes menores (2-5\%) de uma vasta série de compostos químicos. Entretanto, em uma mesma espécie, o conteúdo e a composição do óleo pode variar devido às condições climáticas e agronômicas, qualidade da matéria-prima, método de extração e procedimentos de refino, podendo também sofrer modificações durante o período de estocagem. Na Tabela 1 são apresentadas a composição e a massa molar dos ácidos graxos presentes nos óleos refinados de canola, girassol, milho e soja.

O uso de óleos vegetais na produção de combustíveis alternativos tem sido tema de diversos estudos nas últimas décadas (RAMOS et al., 2003), com destaque na produção de biodiesel (SILVA et al., 2010).

O programa nacional de biodiesel, criado pela lei no 11.097, de 13 de janeiro de 2005 dispõe sobre a introdução do biodiesel na matriz energética brasileira onde fica estabelecido que até 2013 seja obrigatório a adição mínima de 5\% de biodiesel ao óleo diesel comercializado ao consumidor final em todo o território nacional (BRASIL, 2005), criando um mercado de cerca de 2,5 bilhões de litros de biodiesel ao ano, tornando necessário o aumento da oferta global de óleos vegetais nos próximos anos (THOMAZ et al., 2012).

Substituto natural do diesel de petróleo (RAMOS et al., 2003), o biodiesel pode ser definido como um mono-alquil éster de ácidos graxos derivados de fontes renováveis, como óleos vegetais e gorduras animais, obtidos através de um processo de transesterificação (FERRARI et al., 2005). O processo consiste em uma reação de trigliceróis com alcoóis de cadeia curta na presença de um catalisador ácido ou básico (RAMOS et al., 2003), transformando triglicerídeos em moléculas menores de ésteres de ácidos graxos (FERRARI et al., 2005). A reação é limitada pela temperatura de ebulição do álcool e geralmente ocorre entre 313,15 K e 343,15 K (SILVA et al., 2010). Os alcoóis que geralmente são usados na reação de transesterificação são o metanol, etanol, propanol, butanol e amílico, sendo o metanol e etanol usados com maior frequência devido ao seu baixo custo (FUKUDA et al., 2001).

Tabela 1: Composição mássica e massa molar dos ácidos graxos

\begin{tabular}{cccccc}
\hline Ácido graxo & Canola (100.w) & Girassol (100.w) & Milho (100.w) & Soja (100.w) & M (g.mol-1) \\
\hline C14:0 & 0,07 & 0,09 & & 0,09 & 228,38 \\
C16:0 & 4,72 & 6,40 & 12,71 & 11,54 & 256,43 \\
C16:1 & 0,25 & 0,13 & 0,17 & 0,08 & 254,42 \\
C17:0 & & & & 0,05 & 270,45 \\
C17:1 & & 0,04 & & & 268,48 \\
C18:0 & 2,55 & 3,23 & 2,25 & 2,98 & 284,49 \\
C18:1 & 62,56 & 31,89 & 34,96 & 22,91 & 282,47 \\
C18:2 & 20,13 & 56,27 & 47,61 & 55,76 & 280,45 \\
C18:2T & & 0,17 & 0,14 & 0,24 & \\
C18:3 & 7,08 & 0,33 & 0,83 & 5,27 & 278,44 \\
C18:3T & 0,41 & & & 0,55 & \\
C20:0 & 0,62 & 0,27 & 0,54 & 0,25 & 312,54 \\
C20:1 & 1,04 & 0,23 & 0,38 & 0,10 & 310,52 \\
C22:0 & 0,28 & 0,65 & 0,19 & 0,23 & 340,59 \\
C24:0 & 0,17 & 0,25 & 0,22 & & 368,65 \\
C24:1 & 0,12 & & & & 366,63 \\
\hline
\end{tabular}


A transesterificação apresenta um complexo comportamento em que os reagentes (álcool e óleo vegetal), parcialmente miscíveis, formam duas fases em equilíbrio, uma fase superior rica em álcool, onde o catalisador está dissolvido e uma fase inferior rica em óleo vegetal, sendo necessária uma forte agitação para que haja transferência de massa entre as duas fases e consequentemente uma melhor eficiência da reação (ROMERO et al., 2010). O conhecimento do comportamento dessas fases é de grande importância para o processo reativo, pois geram informações que auxiliam na condução da reação e na posterior purificação dos produtos (LANZA et al., 2009). Estudos desta natureza são realizados para diferentes temperaturas em condições de equilíbrio líquido-líquido gerando resultados reprodutíveis e de boa qualidade.

Apesar da importância do assunto, poucas investigações foram realizadas, estudou-se a solubilidade dos óleos de soja, canola e milho em etanol e hexano nas temperaturas de $313 \mathrm{~K}, 318 \mathrm{~K}$ e $328 \mathrm{~K}$ (LANZA et al. 2009), a solubilidade de diferentes óleos vegetais foi determinada em etanol no intervalo de temperatura de 298 a 333 K (ROMERO et al., 2010) e também foi estudada a solubilidade de óleos vegetais em soluções formadas por etanol e água em diferentes temperaturas (SILVA et al., 2010).

Devido à importância do assunto, associado a poucas publicações na literatura, este estudo foi focado na avaliação da solubilidade dos solventes etanol, metanol e isopropanol nos óleos de canola, girassol, milho e soja no intervalo de temperaturas entre $290 \mathrm{~K}$ a $333 \mathrm{~K}$.

\section{Materiais e Métodos}

Para a realização do experimento os óleos vegetais foram adquiridos no comércio local e os alcoóis, todos de pureza analítica, adquiridos da empresa Biotec. Tanto os óleos como os alcoóis foram utilizados sem nenhum tratamento adicional.

Os dados de solubilidade foram determinados utilizando uma célula de vidro encamisada de volume aproximado de $140 \mathrm{~mL}$. Os reagentes (óleo e solvente) foram adicionados no interior da célula de equilíbrio de forma que suas frações mássicas fossem aproximadamente $50 \%$, formando uma mistura parcialmente miscível.

A faixa de temperatura utilizada no experimento foi de 293,15 K a 333,15 K para os sistemas contendo metanol e etanol. Para os sistemas contendo isopropanol foi utilizada a faixa de temperatura de $290,15 \mathrm{~K}$ a 296,15 K devido à elevada solubilidade do isopropanol nos óleos vegetais, mesmo as temperaturas mais próximas da temperatura ambiente.

A temperatura no interior da célula de equilíbrio foi controlada através da utilização de um banho termostático $( \pm 0,5 \mathrm{~K})$ e monitorada com a utilização de um termômetro de bulbo. A mistura foi submetida à forte agitação, utilizando agitador magnético, durante uma hora, e logo após o sistema foi colocado em repouso por período de três horas. Durante o período sem agitação, ocorrem no interior da célula a formação de duas fases distintas, uma fase superior, rica em solvente e uma fase inferior, rica em óleo. Após a separação das fases, uma seringa de vidro conectada a um cateter plástico foi utilizada para coletar $10 \mathrm{~mL}$ da fase rica em óleo e colocada em balão de fundo chato de $250 \mathrm{ml}$ com massa previamente determinada em balança analítica. $\mathrm{O}$ balão juntamente com a amostra teve sua massa novamente determinada e em seguida levado para estufa por 24 horas a 343,15 $\mathrm{K}$ para remoção do solvente solubilizado no óleo. Após a remoção do solvente o balão teve sua massa novamente determinada e dessa forma as massas de óleo vegetal e álcool foram determinados por subtração. Conhecendo-se as massas de óleo $\left(m_{\mathrm{o}}\right)$ e solvente, $\left(m_{\mathrm{a}}\right)$ a composição da fase rica em óleo foi facilmente calculada pelas Equações (1) e (2).

$$
\begin{gathered}
w_{\mathrm{a}}=\frac{m_{\mathrm{a}}}{m_{\mathrm{a}}+m_{\mathrm{o}}} \\
w_{\mathrm{a}}+w_{\mathrm{o}}=1
\end{gathered}
$$

Para os valores de composição de óleo e álcool na fase rica em óleo foram determinado os valores da incerteza experimental (PUGH e WINSLOW, 1966) utilizando como base a Equação (3).

$$
F=F(x, y, \ldots) \rightarrow \delta(F)=\left(\left(\frac{\partial F}{\partial x}\right)^{2} \cdot(\delta x)^{2}+\left(\frac{\partial F}{\partial y}\right)^{2} \cdot(\delta y)^{2}+\ldots\right)^{\frac{1}{2}}
$$

\section{Resultados e Discussões}

Na Tabela 2 são apresentados os dados da solubilidade do metanol nos diferentes óleos estudados em função da temperatura. 
Tabela 2: Solubilidade do metanol $(\boldsymbol{w}$ a) nos diferentes óleos vegetais na faixa de temperatura estudada

\begin{tabular}{|c|c|c|c|c|c|c|}
\hline \multirow{2}{*}{$\mathbf{T}(\mathrm{K})$} & \multicolumn{3}{|c|}{ Canola } & \multicolumn{3}{|c|}{ Girassol } \\
\hline & $100 . w_{0}$ & $100 . w_{a}$ & $\delta$ & $100 . w_{0}$ & $100 . w_{a}$ & $\delta$ \\
\hline 293,15 & 95,437 & 4,563 & $\pm 0,007$ & 95,304 & 4,696 & $\pm 0,007$ \\
\hline 298,15 & 95,703 & 4,297 & $\pm 0,007$ & 95,202 & 4,798 & $\pm 0,008$ \\
\hline 303,15 & 94,524 & 5,476 & $\pm 0,007$ & 94,618 & 5,382 & $\pm 0,009$ \\
\hline 308,15 & 94,074 & 5,926 & $\pm 0,008$ & 94,102 & 5,898 & $\pm 0,007$ \\
\hline 313,15 & 93,843 & 6,157 & $\pm 0,007$ & 93,807 & 6,193 & $\pm 0,007$ \\
\hline 318,15 & 93,569 & 6,431 & $\pm 0,006$ & 93,433 & 6,567 & $\pm 0,007$ \\
\hline 323,15 & 93,074 & 6,926 & $\pm 0,008$ & 92,594 & 7,406 & $\pm 0,008$ \\
\hline 328,15 & 92,399 & 7,601 & $\pm 0,007$ & 92,122 & 7,878 & $\pm 0,008$ \\
\hline 333,15 & 92,264 & 7,736 & $\pm 0,007$ & 91,480 & 8,520 & $\pm 0,007$ \\
\hline \multirow{2}{*}{$T(K)$} & \multicolumn{3}{|c|}{ Milho } & \multicolumn{3}{|c|}{ Soja } \\
\hline & $100 . w_{0}$ & $100 . w_{\mathrm{a}}$ & $\delta$ & $100 . w_{0}$ & $100 . w_{\mathrm{a}}$ & $\delta$ \\
\hline 293,15 & 95,235 & 4,765 & $\pm 0,007$ & 95,105 & 4,895 & $\pm 0,007$ \\
\hline 298,15 & 94,915 & 5,085 & $\pm 0,007$ & 94,624 & 5,376 & $\pm 0,007$ \\
\hline 303,15 & 94,418 & 5,582 & $\pm 0,007$ & 94,087 & 5,913 & $\pm 0,007$ \\
\hline 308,15 & 94,162 & 5,838 & $\pm 0,007$ & 94,013 & 5,987 & $\pm 0,008$ \\
\hline 313,15 & 93,680 & 6,320 & $\pm 0,008$ & 92,996 & 7,004 & $\pm 0,008$ \\
\hline 318,15 & 93,285 & 6,715 & $\pm 0,007$ & 93,294 & 6,706 & $\pm 0,006$ \\
\hline 323,15 & 92,672 & 7,328 & $\pm 0,006$ & 92,463 & 7,537 & $\pm 0,007$ \\
\hline 328,15 & 92,356 & 7,644 & $\pm 0,007$ & 91,993 & 8,007 & $\pm 0,008$ \\
\hline 333,15 & 91,653 & 8,347 & $\pm 0,008$ & 91,276 & 8,724 & $\pm 0,007$ \\
\hline
\end{tabular}

Analisando os valores apresentados na Tabela 2, observa-se um aumento da solubilidade do metanol com o aumento da temperatura em todos os óleos estudados. Os resultados indicam que a solubilidade do metanol é baixa nos óleos estudados, apresentando resultado médio de $4,7 \%$ a $293,15 \mathrm{~K}$ e de $8,3 \%$ a 333,15
K. Esses resultados mostram que em um intervalo de aquecimento de $40{ }^{\circ} \mathrm{C}$ a solubilidade do metanol aumenta apenas $3,6 \%$. Além disso, observa-se que a solubilidade depende da origem vegetal do óleo, apresentando valores crescentes de solubilidade na ordem canola, milho, girassol e soja.

Tabela 3: Solubilidade do etanol $(\boldsymbol{w}$ a) nos diferentes óleos vegetais na faixa de temperatura estudada

\begin{tabular}{ccccccc}
\hline \multirow{2}{*}{ T (K) } & \multicolumn{5}{c}{ Canola } & \multicolumn{3}{c}{ Girassol } \\
\cline { 2 - 7 } & $\mathbf{1 0 0 . w _ { \mathbf { o } }}$ & $\mathbf{1 0 0 . w _ { \mathbf { a } }}$ & $\boldsymbol{\delta}$ & $\mathbf{1 0 0 . w _ { \mathrm { o } }}$ & $\mathbf{1 0 0 . w _ { \mathrm { a } }}$ & $\boldsymbol{\delta}$ \\
\hline 293,15 & 90,135 & 9,865 & $\pm 0,007$ & 90,304 & 9,696 & $\pm 0,007$ \\
298,15 & 88,903 & 11,097 & $\pm 0,007$ & 89,322 & 10,678 & $\pm 0,006$ \\
303,15 & 87,675 & 12,325 & $\pm 0,007$ & 87,709 & 12,291 & $\pm 0,008$ \\
308,15 & 86,421 & 13,579 & $\pm 0,007$ & 86,571 & 13,429 & $\pm 0,007$ \\
313,15 & 85,365 & 14,635 & $\pm 0,007$ & 85,019 & 14,981 & $\pm 0,007$ \\
318,15 & 83,956 & 16,044 & $\pm 0,007$ & 83,214 & 16,786 & $\pm 0,007$ \\
323,15 & 80,838 & 19,162 & $\pm 0,006$ & 81,259 & 18,741 & $\pm 0,007$ \\
328,15 & 78,095 & 21,905 & $\pm 0,006$ & 78,832 & 21,168 & $\pm 0,006$ \\
333,15 & 76,819 & 23,181 & $\pm 0,006$ & 77,016 & 22,984 & $\pm 0,007$ \\
\hline
\end{tabular}


Tabela 3: continuação

\begin{tabular}{ccccccc}
\hline \multirow{2}{*}{ (K) } & \multicolumn{3}{c}{ Milho } & \multicolumn{3}{c}{ Soja } \\
\cline { 2 - 7 } & $\mathbf{1 0 0 . w _ { \mathbf { 0 } }}$ & $\mathbf{1 0 0 . w _ { \mathbf { a } }}$ & $\boldsymbol{\delta}$ & $\mathbf{1 0 0 . w _ { \mathbf { 0 } }}$ & $\mathbf{1 0 0 .}_{\mathbf{a}}$ & $\boldsymbol{\delta}$ \\
\hline 293,15 & 88,619 & 11,381 & $\pm 0,008$ & 88,745 & 11,255 & $\pm 0,007$ \\
298,15 & 88,061 & 11,939 & $\pm 0,007$ & 87,914 & 12,086 & $\pm 0,007$ \\
303,15 & 86,571 & 13,429 & $\pm 0,007$ & 86,222 & 13,778 & $\pm 0,006$ \\
308,15 & 84,817 & 15,183 & $\pm 0,006$ & 84,903 & 15,097 & $\pm 0,008$ \\
313,15 & 83,405 & 16,595 & $\pm 0,007$ & 83,473 & 16,527 & $\pm 0,007$ \\
318,15 & 81,639 & 18,361 & $\pm 0,007$ & 81,820 & 18,180 & $\pm 0,006$ \\
323,15 & 79,585 & 20,415 & $\pm 0,007$ & 79,730 & 20,270 & $\pm 0,007$ \\
328,15 & 76,913 & 23,087 & $\pm 0,006$ & 77,220 & 22,780 & $\pm 0,006$ \\
333,15 & 73,899 & 26,101 & $\pm 0,006$ & 74,720 & 25,280 & $\pm 0,006$ \\
\hline
\end{tabular}

Na Tabela 3 são apresentados os dados da composição mássica da fase rica em óleo para os sistemas pseudobinários estudados formados por etanol e óleo vegetal em função da faixa de temperatura investigada.

Da mesma forma como observado para o metanol, a solubilidade do etanol aumenta com o aumento da temperatura. A solubilidade do etanol é superior a solubilidade do metanol nos óleos estu- dados. Os resultados indicam que a solubilidade do etanol nos óleos e faixa de temperatura avaliada apresenta resultado médio de $10,6 \%$ a $293,15 \mathrm{~K}$ e de $24,4 \%$ a $333,15 \mathrm{~K}$. Esses resultados mostram que em um intervalo de aquecimento de $40{ }^{\circ} \mathrm{C}$ a solubilidade do etanol aumenta 13,8\%. Além disso, como observado com o metanol, a solubilidade depende da origem vegetal do óleo, apresentando valores crescentes de solubilidade na ordem girassol, canola, soja e milho.

Tabela 4: Solubilidade do isopropanol (wa) nos diferentes óleos vegetais na faixa de temperatura estudada

\begin{tabular}{ccccccc}
\hline \multirow{2}{*}{ T (K) } & \multicolumn{3}{c}{ Canola } & \multicolumn{3}{c}{ Girassol } \\
\cline { 2 - 7 } & $\mathbf{1 0 0 . w _ { \mathbf { 0 } }}$ & $\mathbf{1 0 0 . w _ { \mathbf { a } }}$ & $\boldsymbol{\delta}$ & $\mathbf{1 0 0 . w _ { \mathbf { 0 } }}$ & $\mathbf{1 0 0 . w _ { \mathbf { a } }}$ & $\boldsymbol{\delta}$ \\
\hline 290,15 & 72,406 & 27,594 & $\pm 0,006$ & 74,086 & 25,914 & $\pm 0,006$ \\
293,15 & 69,189 & 30,811 & $\pm 0,006$ & 68,123 & 31,877 & $\pm 0,009$ \\
296,15 & 64,217 & 35,783 & $\pm 0,006$ & 62,224 & 37,776 & $\pm 0,005$ \\
\hline \multirow{2}{*}{$\mathbf{T}(\mathbf{K})$} & $\mathbf{M i l h o}$ & & & Soja & \\
\cline { 2 - 7 } & $\mathbf{1 0 0 . w _ { \mathbf { 0 } }}$ & $\mathbf{1 0 0 .} \boldsymbol{w}_{\mathbf{a}}$ & $\boldsymbol{\delta}$ & $\mathbf{1 0 0 .} \boldsymbol{w}_{\mathbf{0}}$ & $\mathbf{1 0 0 . w _ { \mathbf { a } }}$ & $\boldsymbol{\delta}$ \\
\hline 290,15 & 71,142 & 28,858 & $\pm 0,006$ & 71,599 & 28,401 & $\pm 0,007$ \\
293,15 & 66,963 & 33,037 & $\pm 0,005$ & 69,826 & 30,174 & $\pm 0,006$ \\
296,15 & 59,189 & 40,811 & $\pm 0,005$ & 60,629 & 39,371 & $\pm 0,006$ \\
\hline
\end{tabular}

Os resultados do isopropanol apresentados na Tabela 4 mostram como para o metanol e etanol, um aumento da solubilidade do álcool nos óleos com o aumento da temperatura. Todavia a solubilidade do isopropanol nos óleos estudados é muito superior quando comparada com o metanol e com o etanol. Durante os ensaios iniciais foi observado que o isopropanol se solubilizava por completo em todos os óleos investigados a $298,15 \mathrm{~K}$ fazendo com que uma diferente faixa de temperatura fosse avaliada. Para a faixa de temperatura investigada os resulta- dos indicam que a solubilidade do isopropanol nos óleos apresenta resultado médio de $27,7 \%$ a 290,15 K e de $38,4 \%$ a $296,15 \mathrm{~K}$. Esses resultados mostram que em um pequeno intervalo de aquecimento de $6{ }^{\circ} \mathrm{C}$ a solubilidade do isopropanol aumenta $10,7 \%$. 


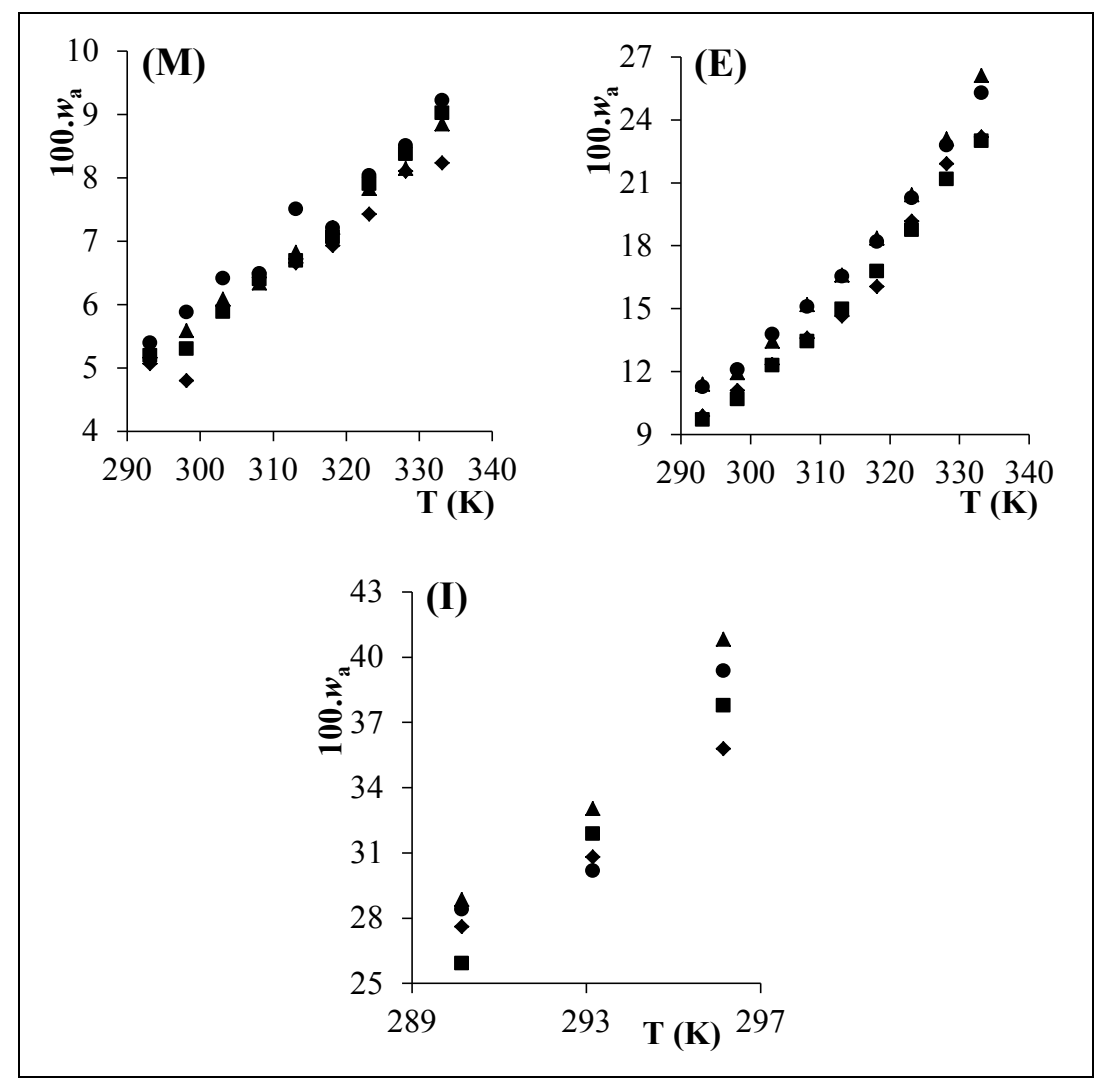

Figura 1: Comportamento da solubilidade do metanol (M), etanol (E) e isopropanol (I) nos óleos estudados em função da temperatura: canola; $\bullet$ girassol; $\boldsymbol{\Delta}$ milho; $\bullet$ soja

Da mesma forma como observado para o metanol e etanol, a solubilidade do isopropanol depende da origem vegetal do óleo, a solubilidade do isopropanol nos óleos analisados cresce na ordem canola, girassol, soja e milho.

Com a finalidade de ilustrar o comportamento dos dados experimentais, a Figura 1 mostra os resultados da solubilidade do metanol, etanol e isopropanol nos diferentes óleos vegetais estudados em função da temperatura.

A afinidade do álcool pelo óleo pode ser interpretada em relação à polaridade das moléculas envolvidas. De maneira geral, os óleos vegetais são formados por moléculas de tamanho relativamente elevado que tendem a ser apolares ou fracamente polares. A constante dielétrica dos constituintes dos óleos vegetais se encontra na faixa entre 2 e 4 (DAMODARAN et al., 2007), de forma que estas moléculas tenham maior afinidade por moléculas também de baixa ou nenhuma polaridade. A polaridade dos alcoóis diminui com o aumento da cadeia carbônica. O metanol, etanol e isopropanol possuem constante dielétrica respectivamente de 32,7, 24,5 e 18,3 de forma que o componente de maior polaridade tem menor afinidade pelo óleo e o componente de menor polaridade tem maior afinidade pelo óleo.

\section{Conclusão}

Metanol, etanol e isopropanol apresentam solubilidade parcial nos óleos de canola, girassol, milho e soja nos intervalos de temperatura investigados. A solubilidade dos alcoóis aumenta com o aumento da temperatura e a quantidade que é solubilizada também depende da natureza do óleo vegetal. Todos os óleos investigados mostram maior afinidade pelos alcoóis com uma relação crescente com o aumento da cadeia carbônica do álcool. Este comportamento apresenta uma dependência da solubilidade com a diminuição da polaridade da molécula do álcool. Devido a esta relação com a polaridade do álcool, o isopropanol apresenta capacidade de se solubilizar nos óleos estudados muito superior a do metanol ou do etanol. 


\section{Referências}

BRASIL; Lei no 11.097- Dispõe sobre a introdução do biodiesel na matriz energética brasileira; altera as Leis nos 9.478, 9.847 e 10.636; e dá outras providências. Diário Oficial da União, Brasília, 2005.

DAMODARAN, S.; PARKIN, K.; FENNEMA, O.R. Fennema's food chemistry, 4th ed., CRC Press, 2007.

FERRARI, R.A.; OLIVEIRA, V.S.; SCABIO, A. Biodiesel de soja - taxa de conversão em ésteres etílicos, caracterização físico-química e consumo em gerador de energia. Química Nova, v. 28, p.1923, 2005.

FUKUDA, H.; KONDO, A.; NODA, H. Biodiesel fuel production by transesterification of oils. Journal of Bioscience and Bioengineering, v. 92, p.405-416, 2001.

LANZA, M.; SANAIOTTI, G.; BATISTA, E.A.C.; POPPI, R.J.; MEIRELLES, A.J.A. Liquid-liquid equilibrium data for systems containing vegetable oils, anhydrous ethanol, and hexane at (313.15, 318.15 and 328.15) K. Journal of Chemical and Engineering Data, v. 54, p.1850-1859, 2009.

PUGH, E.M; WINSLOW, G.H. The analysis of physical measurements, Addison-Wesley, Reading, Mass., 1966.

RAMOS, L.P.; KUCEK, K.T; DOMINGOS, A.K.; WILHELM, H.M. Biodiesel: um projeto de sustentabilidade econômica e sócio-ambiental para o Brasil. Revista Biotecnologia Ciência e Desenvolvimento, v. 31, p.28-37, 2003.

REDA, S.Y.; CARNEIRO, P.I.B. Óleos e gorduras: aplicações e implicações. Revista Analytica, v. 27, p.60-67, 2007.

ROMERO, L.A.F.; LANZA, M.; SILVA, C.A.S.; BATISTA, E.A.C.; MEIRELLES, A.J.A. Mutual solubility of pseudobinary systems containing vegetable oils and anhydrous ethanol from (298.15 to 333.15) K. Journal of Chemical and Engineering Data, v. 55, p.2750-2756, 2010.
SILVA, C.A.S.; SANAIOTTI, G.; LANZA, M.; ROMERO, L.A.F.; MEIRELLES, A.J.A.;

BATISTA, E.A.C. Mutual solubility for systems composed of vegetable oil + ethanol + water at different temperatures. Journal of Chemical and Engineering Data, v. 55, p.440-447, 2010.

THOMAZ, G.L.; ZAGONEL, J.L.; COLASANTE, O.; NOGUEIRA, R.R. Produção do girassol e teor de óleo nas sementes em diferentes épocas de semeadura no centro-sul do Paraná. Ciência Rural, v. 42, p.203-208, 2012. 